\title{
Immunological biomarkers of the vitreous responsible for proliferative alteration in the different forms of retinal detachment
}

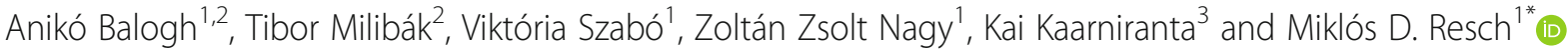

\begin{abstract}
Background: The purpose of the study was to explore the immunological components that are responsible for the proliferative alterations in the different forms of retinal detachment (RD).

Methods: Vitreous fluids were collected during 23G pars plana vitrectomy from 54 eyes of 54 patients with different RD types, such as rhegmatogenous RD (RRD) without proliferative vitreoretinopathy (PVR) $(n=30)$, PVR $(n=16)$ and proliferative diabetic retinopathy (PDR) with tractional RD $(n=8)$. Vitreous fluids were obtained from 19 eyes with epiretinal membrane (ERM), which were used as control samples. A multiplex chemiluminescent immunoassay was performed to evaluate the concentrations of 48 cytokines, chemokines and growth factors.

Results: The expression levels of eotaxin, IFN-gamma, IL-6, IL-8, IL-16, MCP-1, MIF and MIP-1 beta were significantly higher in all RD groups than in the ERM group. The levels of CTACK, IP-10, SCGF-beta, and SDF-1 alpha were significantly higher in patients with diabetic tractional RD and PVR than in other patients. The upregulation of VEGF and IL-18 was detected in PDR.

Conclusions: Our results indicate that complex and significant immunological mechanisms are associated with the pathogenesis of different forms of RD: selected cytokines, chemokines and growth factors are upregulated in the vitreous of eyes with RD. The detected proteins are present in different concentrations both in RRD and PVR. In the presence of PVR and PDR, the majority of cytokines are upregulated; thus, they may serve as biomarkers to estimate the progression or severity level of proliferation and later to develop personalized therapeutic strategies to slow down or prevent pathological changes.
\end{abstract}

Keywords: Cytokine, Chemokine, Vitreous, Proliferative vitreoretinopathy, Retinal detachment, Proliferative diabetic retinopathy

\section{Background}

Retinal detachment (RD) can cause vision loss if untreated, and even with proper surgical intervention, a potentially sight-threatening condition may develop in some cases. The most difficult challenges for vitreoretinal surgeons are proliferative diabetic retinopathy (PDR) complicated with

\footnotetext{
* Correspondence: miklosresch@gmail.com

'Department of Ophthalmology, Semmelweis University, Mária u 39, Budapest 1085, Hungary

Full list of author information is available at the end of the article
}

tractional RD and proliferative vitreoretinopathy (PVR) developed from rhegmatogenous RD (RRD).

PVR is based on the development of fibrocellular membranes on the surface of and under the retina after RRD [1]. PDR is characterized by neovascularization on the retina and the formation of fibrovascular membranes at the vitreoretinal interface. The development of fibrovascular tissue often leads to hemorrhage and tractional RD [2].

According to our current knowledge, there is no cure or prophylaxis available for PVR yet, apart from the 
surgical approach [3]. In the treatment of PDR, pars plana vitrectomy plays the main role with various microsurgical techniques. Iyer et al. proposed a surgical algorithm for the management of PDR with tractional RD based on their compilation of relevant literature [4]. Because of these difficulties, the pathophysiology of PVR and PDR, including cytokines, chemokines and other inflammatory factors, is under investigation [5].

Many studies reported an immunological component responsible for PVR and the formation of tractional RD in PDR. In the first studies, only a few proteins could be assayed in one sample by enzyme-linked immunosorbent assay (ELISA) [6-8]. Caepaens et al. were among the first to evaluate three chemokines with ELISA in vitreous samples and found that the MCP-1 level was significantly higher in PVR and PDR samples than in controls [9].

Currently, a new technique, the multiplex bead-based immunoassay, provides an opportunity to perform a wide range of molecular analyses in one sample. This helps us understand the interaction among the components of the immunological processes responsible for pathological changes in PDR and PVR [10, 11]. Clinical evidence comparing intraocular cytokine, chemokine and growth factor levels in patients with PVR, PDR and RRD is scarce. Understanding the role of immunological factors in the pathophysiology of different RDs is important to be able to develop new therapeutic targets.

The purpose of this study was to explore the immunological components that are responsible for the proliferative alterations in PVR and PDR and to gain more detailed information and compare the differences in the levels of cytokines, chemokines and growth factors in the vitreous among the different forms of RD.

\section{Methods}

The project was performed under the tenets of the Declaration of Helsinki after approval by the Hungarian Medical Research Council Committee of Science and Research Ethics (No. 15028-2/2017/EKU). All participants gave written informed consent to participate in the study.

Seventy-three eyes of 73 patients undergoing pars plana vitrectomy were included in the cross-sectional study. Patients were divided into four groups according to the indicated ocular pathology: 30 patients with RRD (without PVR), 16 patients with PVR, 8 patients with PDR and 19 control patients with idiopathic epiretinal membrane (ERM). The exclusion criteria were previous vitreoretinal surgery, penetrating injury, uveitis, aphakia, age-related macular degeneration, and uncontrolled glaucoma. Diabetes mellitus was excluded in the RRD, PVR and ERM groups. In the PDR group, only wellcontrolled diabetic patients (with glycated hemoglobin [HbA1c] under 8\%) were included, and pure tractional
$\mathrm{RD}$ was present (without vitreous hemorrhage or active proliferation). Previous panretinal photocoagulation was permitted, but intravitreal treatments (steroid or antiVEGF) were excluded. A priori sample size calculation (power $=0.90 ; p=0.05$ ) was performed and provided the minimum number of eyes to be 65 eyes. The vitreous samples of patients with diabetic retinopathy, RD and ERM were collected for 2 years. As a limited number of samples can be analyzed on one kit of the multiplex chemiluminescent immunoassay, we had to define the maximum number of vitreous fluids. After we analyzed the patients' data, we excluded the samples that were not matched the standing criteria. We had to exclude a lot of the diabetic samples because of previous intravitreal injections, active proliferation and high glycated hemoglobin.

Demographic and clinical data are summarized in Table 1.

\section{Sample collection}

Pars plana vitrectomy (23G) was performed by two surgeons in two vitreoretinal centers. Before starting irrigation $0.5 \mathrm{ml}$ undiluted vitreous samples were collected from the eyes. The samples were injected into sterile Eppendorf tubes, immediately cooled to $-20^{\circ} \mathrm{C}$ then frozen at $-80^{\circ} \mathrm{C}$ within $2 \mathrm{~h}$ and stored until the assay was performed.

\section{Measurement of cytokines, chemokines and growth factors in vitreous samples}

The concentrations of cytokines, chemokines, and growth factors in vitreous samples were measured with a multiplex bead-based immunoassay: Bio-Plex system (Bio-Rad Laboratories, Hercules, CA, USA), molecules were detected with the Human Cytokine Screening Panel, 48-Plex (Bio-Rad Laboratories) after fourfold dilution. According to relevant previous works [12, 13]. The detailed specifications of the sample preparation were mentioned in our previous article [14]. Measurement of 84 molecules using 96-well assay plates and reagent kits included (see abbreviations): CTACK, eotaxin, basic FGF, G-CSF, GM-CSF, GRO-alpha, HGF, IFN-alpha2, IFN-gamma, IL-1 alpha, IL-1 beta, IL-1ra, IL-2, IL-2R alpha, IL-3, IL-4, IL-5, IL -6, IL-7, IL-8, IL-9, IL-10, IL12/p40, IL-12/p70, IL-13, IL-15, IL-16, IL-17, IL-18, IP-10, LIF, MCP-1, MCP-3, M-CSF, MIF, MIG, MIP-1 alpha, MIP1 beta, beta-NGF, PDGF-BB, RANTES, SCF, SDF-1 alpha, SCGF-beta, TNF-alpha, TNF-beta, TRAIL and VEGF were analyzed. If concentrations of the molecules were too low or too high, output was given as $<\mathrm{OOR}$ or $>\mathrm{OOR}$ meaning below or over the range (out of range).

\section{Statistical analysis}

Cytokine concentrations were analyzed with, KruskalWallis analysis of variance and Dunn's multiple 
Table 1 Demographic and clinical data of patients. Age, symptom duration and extent of RD are given in Mean \pm SD. RRD rhegmatogenous retinal detachment, PVR - proliferative vitreoretinopathy, PDR - proliferative diabetic retinopathy, ERM - Epiretinal membrane

\begin{tabular}{|c|c|c|c|c|c|}
\hline & & RRD & PVR & PDR & ERM \\
\hline $\mathrm{N}$ (male/female) & & $30(18 / 12)$ & $16(8 / 8)$ & $8(5 / 3)$ & $19(5 / 14)$ \\
\hline Age (years) & & $61(7,5)$ & $58,4(11,9)$ & $55(9,7)$ & $70,7(8,9)$ \\
\hline Symptom duration (days) & & $7.0 \pm 6.4$ & $30.2 \pm 28.3$ & $43.4 \pm 15.0$ & NA \\
\hline Macula on/off & & $13 / 17$ & $3 / 13$ & $2 / 6$ & NA \\
\hline Extent of RD (quadrants) & & $1.9 \pm 0.7$ & $2.9 \pm 0.9$ & $2.8 \pm 0.8$ & NA \\
\hline \multirow[t]{4}{*}{ Location of tears (\%) } & Superior & 50 & 31.2 & NA & NA \\
\hline & Inferior & 6.6 & 56.3 & NA & NA \\
\hline & Temporal & 36.7 & 0 & NA & NA \\
\hline & Nasal & 6.6 & 12.5 & NA & NA \\
\hline \multirow[t]{3}{*}{ Endotamponade (\%) } & SF6 gas & 10 & 18.7 & 12.5 & 47.3 \\
\hline & C3F8 gas & 73.3 & 50 & 50 & 52.7 \\
\hline & Silicone oil & 16.7 & 31.3 & 37.5 & 0 \\
\hline
\end{tabular}

comparison test. $P$-values were calculated via dedicated statistical software (GraphPad Prism, La Jolla, CA). Pvalues $<0.05$ were set to indicate statistical significance.

Exploratory factor analysis. Since not all correlations are well known among the examined cytokines, exploratory factor analysis was performed with SPSS 23.0 software (IBM, Chicago, IL, USA).

\section{Results}

An assay could be performed on all samples. Table 2 lists the median and SD of all individual cytokines in the four patient groups. The Kruskal-Wallis test selected 18 out of 48 cytokines that reached the level of significance in concentration (Table 3). The most important dependent variables are highlighted below in Figs. 1, 2, 3, 4.

Seven cytokines were upregulated in all $R D$ groups $(R R D$, PVR and PDR) compared to controls: levels of IL-6 ( $p<$ $0.001, p<0.001$ and $p<0.001$, respectively), IL-16 $(p<0.01$, $p<0.001$ and $p<0.001$, respectively), IFN-gamma $(p<0.001$, $p<0.001$ and $p<0.05$, respectively), MCP-1 $(p<0.001, p<$ 0.001 and $p<0.01$, respectively), and MIF ( $p<0.001, p<$ 0.001 and $p<0.001$, respectively) were significantly higher in all RD groups than in the ERM group. The concentrations of IL-8 $(p<0.01, p<0.001$ and $p<0.01$, respectively $)$ and eotaxin $(p<0.05, p<0.001$ and $p<0.01$, respectively) were significantly higher in PVR and PDR than in ERM and significantly lower in RRD than in PDR (Fig. 1). Further comparisons between groups are summarized in Table 3 .

There were four upregulated cytokines in the PDR and PVR groups compared to the RRD and ERM groups (Fig. 2): CTACK was highly upregulated in patients with PVR $(p<$ 0.05 PVR vs RRD) and PDR ( $p<0.01$ PDR vs ERM; $p<$ 0.001 PDR vs RRD). Levels of IP-10 were upregulated in PDR and PVR vs ERM $(p<0.001$ both) and upregulated in PDR vs RRD $(p<0.05)$; however, they were not different in
PVR vs RRD. SCGF-beta exhibited the highest expression levels in PVR ( $p<0.05$ PVR vs RRD) but were not different in PDR vs ERM or RRD. SDF1-alpha was prominent in the PVR ( $p<0.001$ PVR vs ERM; $p<0.01$ PVR vs RRD) and the PDR ( $p<0.001$ PDR vs ERM; $p<0.01$ PDR vs RRD) groups.

The concentration values of VEGF in the vitreous fluid were significantly higher in the PDR group than in other groups $(p<0.05$ PDR vs ERM; $p<0.001$ PDR vs RRD and $p<0.01$ PDR vs PVR). The vitreous level of IL-18 was found to be elevated in the PDR group compared to $\operatorname{ERM}(p<0.01)$ and RRD $(p<0.05)$ (Fig. 3$)$.

Levels of IL-2R alpha $(p<0.05)$, IL-17 $(p<0.05)$ and HGF $(p<0.05)$ were significantly higher in PDR than in $R R D$. The concentration of beta-NGF was significantly elevated in PDR compared to RRD $(p<0.05)$ and PVR $(p<$ $0.05)$. The levels of MIG were significantly higher in PDR $(p<0.01)$ and ERM $(p<0.001)$ than in RRD (Fig. 4).

\section{Exploratory factor analysis}

As the first step of exploratory factor analysis, variables (molecules) were filtered. Only 29 out of 48 molecules were included in the factor analysis, when valid concentration data were available for at least 70 out of 73 patients. Pearson correlation analysis revealed that a significant correlation was found among all 29 variables. The requirements of exploratory factor analysis were fulfilled: the communality value was higher than 0.3 among all variables, and the Kaiser-Meyer-Olkin (KMO) value was 0.833 . The explanatory power of the 6 new variables was $82.36 \%$; therefore, the dimension reductive method could be performed. The 29 variables could be grouped into 6 dimensions:

Dimension 1: IL-15, IL-2, Basic FGF, IL-1 beta, IL-12 (p40), IL-7, IL-4, IL-17, RANTES, IL-5, IL-13 and GRO-alpha. 
Table 2 The concentration of cytokines in the groups in pg/ml (median; min.; max.)

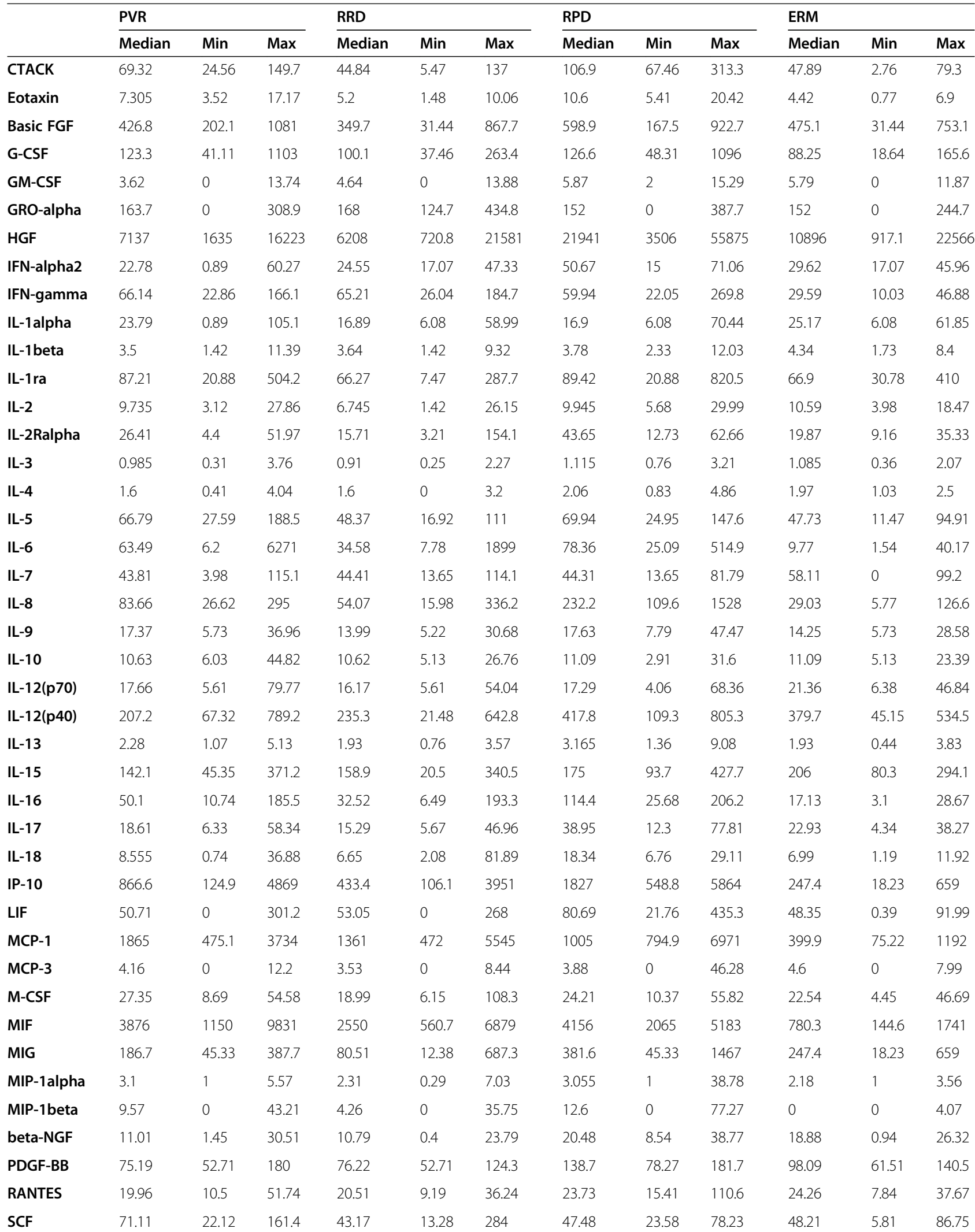


Table 2 The concentration of cytokines in the groups in pg/ml (median; min.; max.) (Continued)

\begin{tabular}{|c|c|c|c|c|c|c|c|c|c|c|c|c|}
\hline & \multicolumn{3}{|l|}{ PVR } & \multicolumn{3}{|l|}{ RRD } & \multicolumn{3}{|l|}{ RPD } & \multicolumn{3}{|l|}{ ERM } \\
\hline & $\overline{\text { Median }}$ & Min & Max & $\overline{\text { Median }}$ & Min & Max & $\overline{\text { Median }}$ & Min & Max & $\overline{\text { Median }}$ & Min & Max \\
\hline SCGF-beta & 28963 & 3532 & 76005 & 10997 & 1684 & 295273 & 22418 & 3481 & 141845 & 11256 & 736.6 & 37990 \\
\hline SDF-1alpha & 209.6 & 53.4 & 381.2 & 81.34 & 0 & 430.5 & 214.5 & 90.99 & 476.8 & 70.11 & 0 & 139 \\
\hline TNF-alpha & 21.79 & 3.69 & 94.43 & 27.08 & 0 & 55.58 & 32.32 & 11.07 & 113.6 & 28.83 & 12.87 & 54.73 \\
\hline TNF-beta & 6.73 & 0 & 45.18 & 5.52 & 0 & 30.32 & 9.7 & 0 & 38.86 & 7.93 & 0 & 27.07 \\
\hline TRAIL & 13.45 & 3.44 & 29.53 & 13.19 & 6.78 & 25.51 & 18.38 & 5.68 & 112.4 & 10.01 & 0 & 21.96 \\
\hline VEGF & 225 & 87.55 & 553.5 & 244.7 & 63.84 & 500.5 & 614.4 & 255.8 & 4289 & 272.1 & 87.55 & 443.4 \\
\hline
\end{tabular}

Dimension 2: SCGF-beta, SCF, IL-2R alpha and M-CSF. Dimension 3: MIP-1 beta, MCP-3, IL-1ra, MIG and IL-8. Dimension 4: Eotaxin and CTACK

Dimension 5: IL-18, MIF and IL-16

Dimension 6: IFN-alpha2, PDGF-BB and TRAIL

Dimensions mean that the molecules within one dimension exhibit significant activity as individual biomarkers, and their changes are correlated.

Factor weights are summarized in Table 4, which shows that there is no difference among groups in dimensions 1 and 2, but significant differences were found in dimensions 3-6 (Fig. 5).

\section{Discussion}

Our results show that common vitreous biomarkers involved in RDs are IL-6, IL-16, IFN-gamma, MCP-1 and MIF. This finding reveals the strong inflammatory component in the pathology of RD. However, different RD types show a phenotype-dependent profile in the expression of cytokines.

The interpretation of our data is challenging due to the complexity of the molecules and RD pathomechanism. Previous studies analyzed similar methods but different aspects of RD. Wladis et al. documented that IL4, IL-5, IL-6, IL-15, G-CSF, GM-CSF, IP-10, MIP-1 alpha, SCF, and SCGF were significantly increased in PVR compared to primary RRD and ERM [15]. The upregulation of IP-10 and SCGF is in line with our observations. It seems that chemoattraction plays a central role in the pathogenesis of PVR when IL-8 and IP-10 are used as biomarkers. Upregulation of IL-6 and SCGF revealed that our PVR samples represent a late-state process with chronic inflammation and fixed retinal folds [16]. At present, there is no cure or effective prophylaxis for PVR. Autophagy regulators are effective

Table 3 Cytokines with significant difference in case of RD. ${ }^{*} p<0.05$; ${ }^{* *} p<0.01$; ${ }^{* *} p<0.001$

\begin{tabular}{|c|c|c|c|c|c|c|}
\hline & RRD > ERM & PVR $>$ ERM & PDR $>$ ERM & PVR $>$ RRD & PDR $>$ RRD & PDR $>$ PVR \\
\hline IL-6 & $* * *$ & $* * *$ & $* * *$ & & & \\
\hline IL-16 & $* *$ & $* * *$ & $* * *$ & & & \\
\hline IFN-gamma & $* * *$ & $* * *$ & * & & & \\
\hline MCP-1 & $* * *$ & $* * *$ & $* *$ & & & \\
\hline MIF & $* * *$ & $* * *$ & $* * *$ & & & \\
\hline IL-8 & & $* *$ & $* * *$ & & $* *$ & \\
\hline eotaxin & & * & $* * *$ & & $* *$ & \\
\hline CTACK & & & $* *$ & * & $* * *$ & \\
\hline IP-10 & & $* * *$ & $* * *$ & & $*$ & \\
\hline SCGF-beta & & & & * & & \\
\hline SDF-1alpha & & $* * *$ & $* * *$ & $* *$ & $* *$ & \\
\hline VEGF & & & * & & $* * *$ & $* *$ \\
\hline IL-18 & & & $* *$ & & $*$ & \\
\hline IL-2Ralpha & & & & & * & \\
\hline IL-17 & & & & & * & \\
\hline HGF & & & & & * & \\
\hline Beta-NGF & & & & & $*$ & $*$ \\
\hline MIG & & & & & $* *$ & \\
\hline
\end{tabular}



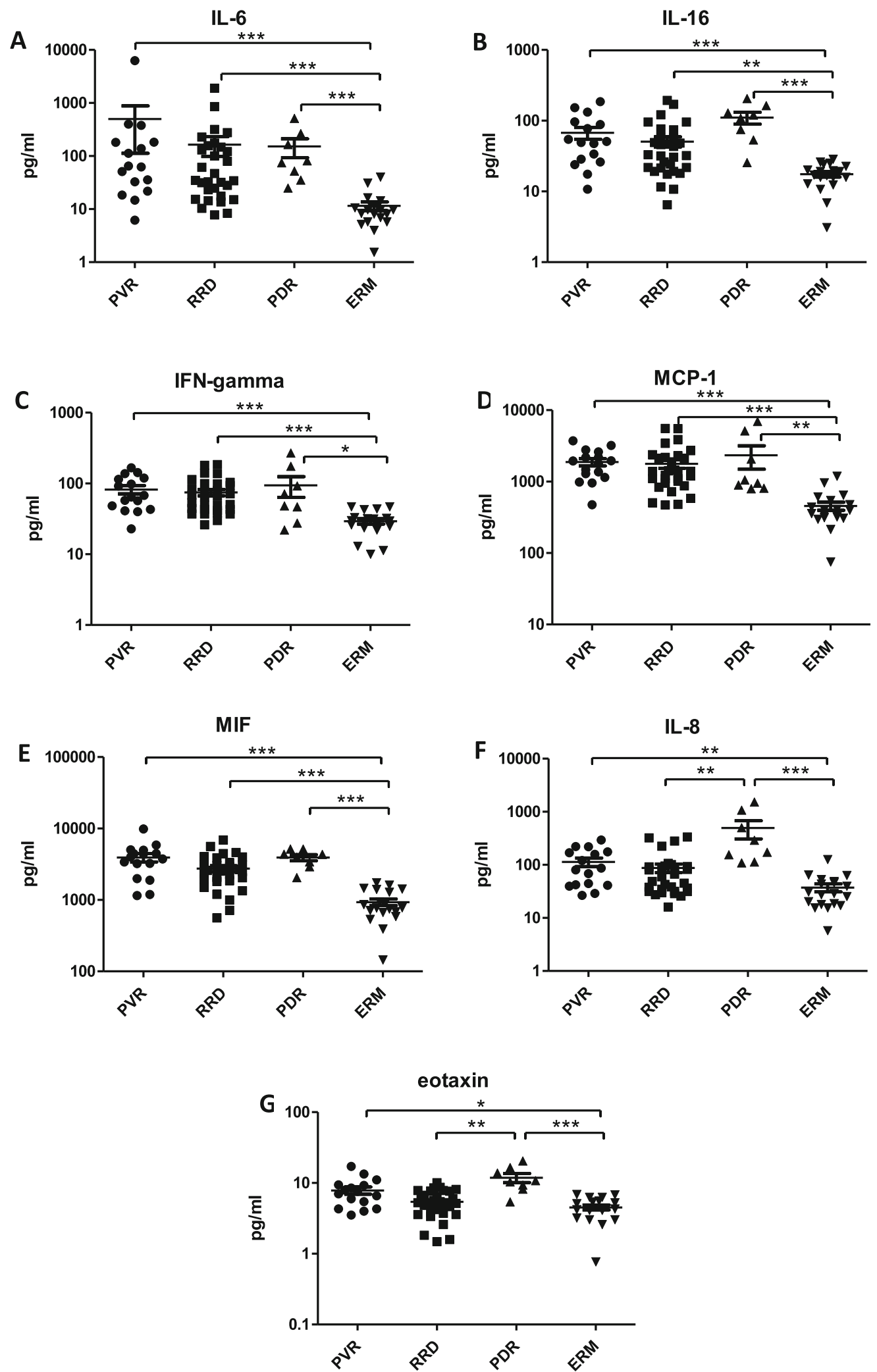

Fig. 1 Upregulated molecules in PVR, RRD and PDR compared to ERM. Concentrations of IL-6, - 16, IFN-gamma, MCP-1, MIF, IL-8 and eotaxin in eyes with PVR, RRD, PDR and ERM. Statistically significant differences between the groups are marked by asterisks. ${ }^{*} p<0.05 ;{ }^{* *} p<0.01 ;{ }^{* * *} p<0.001$ 

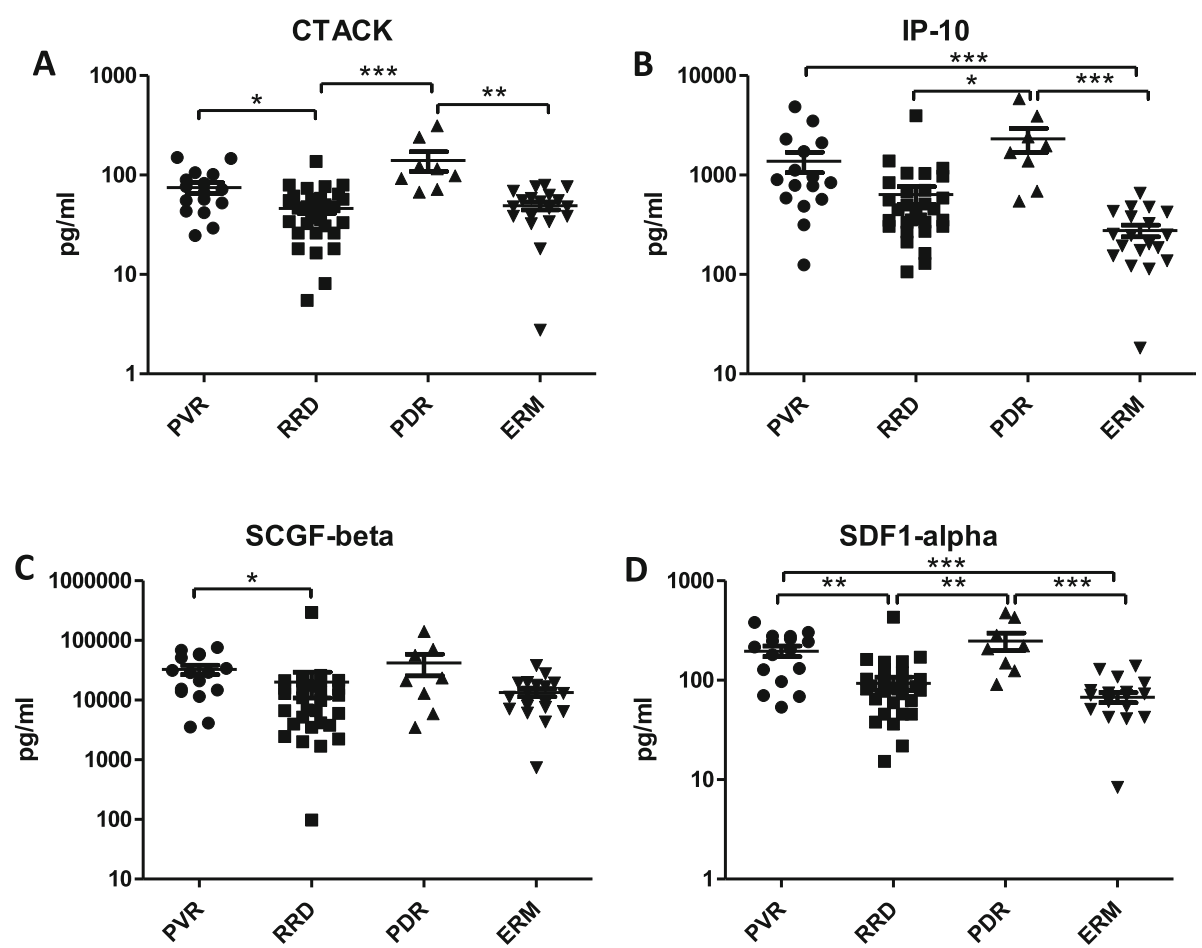

Fig. 2 Upregulated molecules in PVR and PDR compared to RRD and ERM. Concentrations of CTACK, IP-10, SCGF-beta and SDF1-alpha in eyes with PVR, RRD, RPD and ERM. Statistically significant differences between the groups are marked by asterisks. ${ }^{*} p<0.05$; ${ }^{* *} p<0.01$; ${ }^{* * *} p<0.001$

in the prevention of PVR in animal models [17]. Pennock et al. suggested that ranibizumab might potentially prevent PVR formation since it reduces the bioactivity of the vitreous both in experimental animal models and humans. Ranibizumab prevented the growth of PVR membranes in rabbits as well [18]. In a mouse model, IL-6 receptor blockers have been shown to reduce retinal fibrosis [19]. Kawahara et al. proposed that statins might be potent inhibitors of cicatricial contraction in proliferative vitreoretinal diseases. In their paper they publsihed that in an experimental model the intravitreal injection of simvastatin dose-dependently prevented the progression of PVR [20]. Since acknowledgments of cellular signaling mechanisms have improved in recent years, we can develop specific targeted therapies to prevent PVR. For that purpose, patient material use in translational medicine research is crucial.

Abu El-Asrar et al. evaluated the levels of ten chemokines with ELISA in the vitreous from eyes with RRD, PVR, and PDR, and they came to the conclusion that MCP-1, IP-10, and SDF-1 might be factors in the pathogenesis of PVR and PDR. Our results are consistent with Abu El-Asrar's, but we could analyze a wider range of molecules in each sample with the help of a multiplex bead-based immunoassay [8]. Wang et al. showed that levels of IL-6 and MCP-1 were significantly higher in the vitreous and aqueous humor in patients with PDR than in controls with macular hole (MH) [21]. Dai et al.
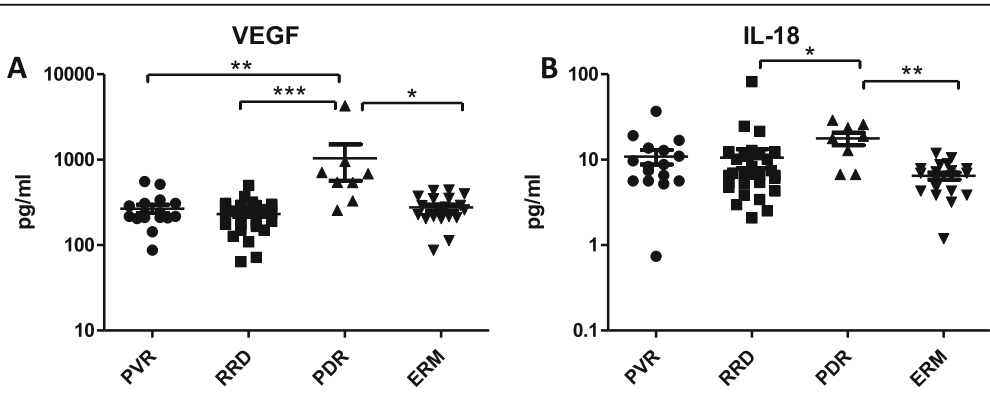

Fig. 3 Upregulated molecules in PDR compared to PVR, RRD and ERM. Concentrations of VEGF and IL-18 in eyes with PVR, RRD, RPD and ERM. Statistically significant differences between the groups are marked by asterisks. ${ }^{*} p<0.05 ;{ }^{* *} p<0.01 ;{ }^{* *} p<0.001$ 


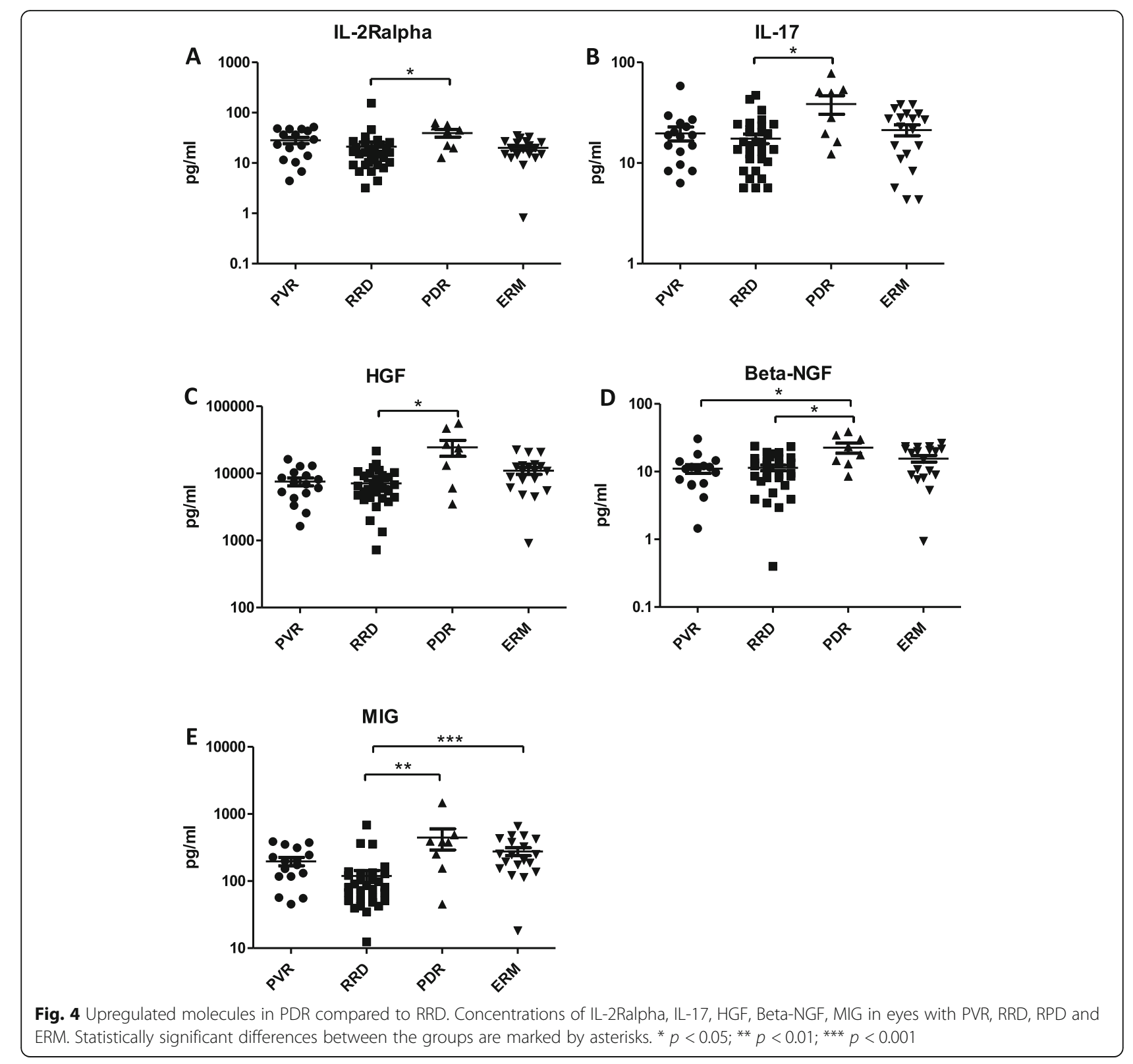

documented that MCP-1, MIP-1 beta, IP-10, MIG and VEGF levels were increased in PDR compared to ERM and $\mathrm{MH}$ [22]. The same proteins were upregulated in the vitreous of our PDR samples. Moreover, CTACK and eotaxin levels were prominent in our vitreous samples. In PDR pathology, chemoattraction seems to be active, but as a disease characteristic sign, increased angiogenesis through VEGF can be observed, as previously shown. Note that elevated VEGF levels were not detected in PVR pathogenesis. The role of VEGF in diabetic macular edema and PDR is well known [23]. We observed that the levels of IL-18 and VEGF were significantly higher in PDR than in other conditions. Song et al. documented that the levels of intravitreal VEGF and IL-18 were significantly higher in active PDR than in ERM and $\mathrm{MH}$ [24]. Xu et al. found that the vitreous levels of CCL2, CXCL4, CXCL9, CXCL10, VEGF, sVEGFR-1, sVEGFR-2, IL-6, IL-8, IL-10, and IL-18 were elevated significantly in the PDR group compared to the nondiabetic group [25]. In our study, we found significant upregulation in the levels of IL-18 in PDR compared to the control group and RRD separately. Increased IL-18 expression levels in vitreous fluid reveal inflammasome activation [26]. Inflammasomes are large cytosolic protein complexes composed of Nod-like receptor sensor protein, adaptor protein ASC and caspase, mainly caspase-1, as an effector enzyme [27]. Inflammasome activation results in the release of the 
Table 4 Explorative factor analysis: Factor weights are summarized in the table, which shows that there is no difference among groups in dimensions 1 and 2, but significant differences were found in dimensions 3-6

\begin{tabular}{|c|c|c|c|c|c|c|c|}
\hline & & \multicolumn{6}{|c|}{ Component } \\
\hline & & 1 & 2 & 3 & 4 & 5 & 6 \\
\hline \multirow[t]{12}{*}{ Dimension 1} & IL-15 & 0.915 & & & & & \\
\hline & $\mathbb{I L}-2$ & 0.883 & & & & & \\
\hline & Basic FGF & 0.881 & & & & & \\
\hline & IL-1 beta & 0.866 & & & & & \\
\hline & IL-12(p40) & 0.840 & & & & & \\
\hline & $\mathrm{IL}-7$ & 0.814 & & & & & \\
\hline & $\mid \mathrm{L}-4$ & 0.765 & & & & & \\
\hline & $\mathbb{I L}-17$ & 0.759 & & & & & \\
\hline & RANTES & 0.730 & & & & & \\
\hline & $\mathbb{I L}-5$ & 0.678 & & & & & \\
\hline & $\mathbb{L L}-13$ & 0.626 & & & & & \\
\hline & GRO-alpha & 0.494 & & & & & \\
\hline \multirow[t]{4}{*}{ Dimension 2} & SCGF-beta & -0.001 & 0.906 & & & & \\
\hline & SCF & 0.234 & 0.876 & & & & \\
\hline & IL-2Ralpha & 0.327 & 0.841 & & & & \\
\hline & M-CSF & 0.507 & 0.761 & & & & \\
\hline \multirow[t]{5}{*}{ Dimension 3} & MIP-1beta & 0.035 & 0.258 & 0.862 & & & \\
\hline & MCP-3 & 0.402 & 0.036 & 0.834 & & & \\
\hline & IL-1 ra & 0.252 & 0.053 & 0.783 & & & \\
\hline & MÍG & 0.211 & 0.451 & 0.687 & & & \\
\hline & IL-8 & 0.052 & -0.095 & 0.602 & & & \\
\hline \multirow[t]{2}{*}{ Dimension 4} & Eotaxin & 0.233 & 0.130 & 0.014 & 0.812 & & \\
\hline & CTACK & 0.252 & 0.444 & 0.056 & 0.699 & & \\
\hline \multirow[t]{3}{*}{ Dimension 5} & $\mathbb{I L}-18$ & 0.132 & 0.209 & 0.098 & 0.033 & 0.824 & \\
\hline & MIF & -0.066 & 0.315 & 0.164 & 0.363 & 0.770 & \\
\hline & IL-16 & -0.119 & 0.427 & 0.311 & 0.344 & 0.675 & \\
\hline \multirow[t]{3}{*}{ Dimension 6} & IFN-alpha2 & 0.466 & 0.043 & 0.148 & 0.244 & 0.057 & 0.727 \\
\hline & PDGF-BB & 0.486 & -0.037 & 0.228 & -0.131 & 0.168 & 0.669 \\
\hline & TRAIL & 0.249 & 0.092 & -0.012 & 0.532 & 0.098 & 0.639 \\
\hline
\end{tabular}

proinflammatory cytokines IL-1 beta and IL-18. Since inflammasomes seem to be activated during the late state of the DR process, they might be a good therapeutic target to prevent tractional $\mathrm{RD}$ once there is no response to current therapy drugs.

Takahashi et al. documented increased levels of IL-6, IL-8, IP-10 and MCP-1 in RRD compared to the control $\mathrm{MH}$ group [12]. These results are similar in our study when ERM cases were used as controls. They also found higher IL-6 and IL-8 levels, but not MCP-1 and IP-10, in RRD than in PDR. Our study showed higher IL-8 and MCP-1 levels in PDR than in RRD. This reveals a stronger chemoattraction in PDR with tractional detachment. Similar to our study, Pollreisz et al. found that IL-6, IL-8 and MCP-1 were elevated in the vitreous of RRD eyes compared to ERM. In contrast to our results, they observed an increase in IP-10 and MIP-1 alpha in RRD [28]. Rasier et al. found that the levels of IL-8 and VEGF were elevated in vitreous samples in eyes with RRD compared to samples from eyes with ERM and MH [29]. Ricker et al. reported that the concentrations of IL-1 alpha, IL-2, IL-3, IL-6, VEGF and ICAM are increased in the subretinal fluid of PVR but not in RRD [30]. We show here that the expression of VEGF was significantly higher only in the PDR group than in the RRD, PVR and ERM groups. Our results are consistent with previous reports $[12,15]$. It seems that VEGF has the strongest biomarker role in PDR with and without tractional detachment. Interestingly, the levels of CTACK, IP-10 and SDF1-alpha were significantly higher in PVR and PDR 


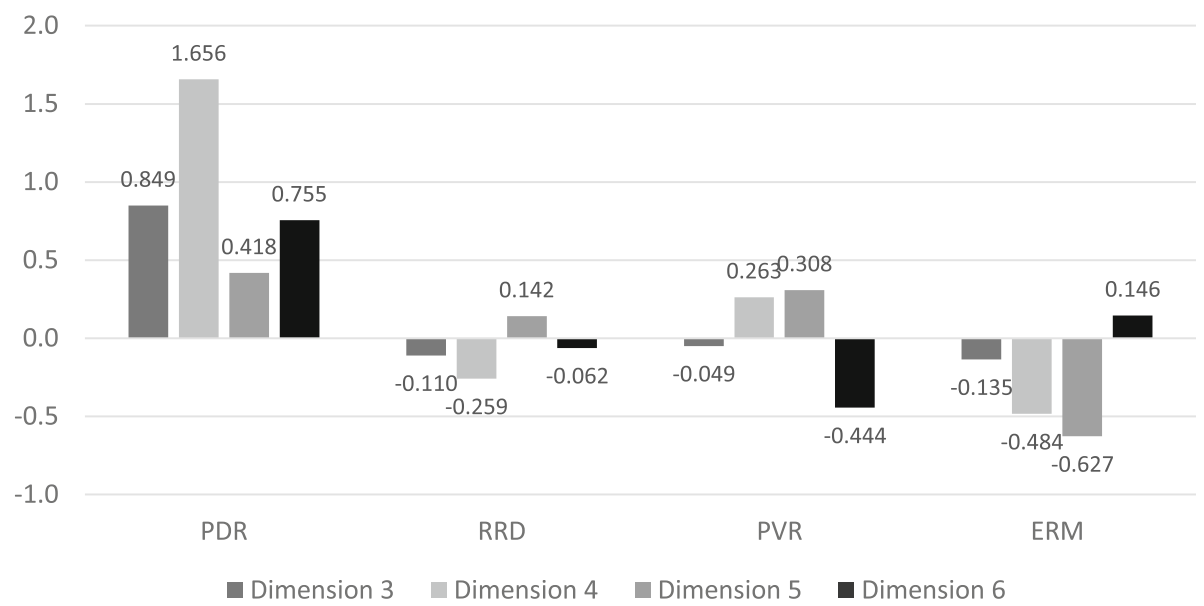

Fig. 5 Exploratory factor analysis. Dimension 3: MIP-1 beta, MCP-3, IL-1ra, MIG, IL-8; Dimension 4: eotaxin, CTACK; Dimension 5: IL-18, MIF, IL-16; Dimension 6: IFN-alpha2, PDGF-BB, TRAIL

than in RRD, while the stem cell factor SCGF had a stronger presence in PVR than in RRD. The concentration of these four chemokines was augmented only in the proliferative forms of RD. CTACK, IP-10 and SDF-1 play a role in a wide variety of processes, such as chemotaxis, immune response, cell-cell signaling, differentiation, activation of peripheral immune cells, and regulation of endothelial cell proliferation.

When we obtain the result of factor analysis, we focus on which factor and variable coheres with each other. At the same time, we can evaluate the factor weights of the variables to be able to hierarchize the variables of one factor. The bigger the factor weight the more important the variable of one factor, so we can analyze separately how the factor weight develops in a given factor. For example in the group of factor 3 MIP-1beta was the most important element, it had a bigger factor weight than IL-8 (Table 4).

To our knowledge, our report is the first to evaluate the concentrations of these 48 cytokines, chemokines, and growth factors in different forms of retinal detachment, including RRD, PVR and PDR with tractional RD. However, our study has some limitations, such as the complexity and high number of cytokines, that need further investigation to detect their relationships more precisely. RD and PDR present with variable clinical features, which might contribute to the multiplex variations in cytokines in fluids. In addition, it cannot be identified whether cytokines are upregulated in the vitreous due to RD (as a consequence) or are already present before detachment (as a causative agent). This limitation is hard to solve due to ethical reasons since the vitreous of healthy human eyes is not accessible in everyday routine clinical care. The exploratory factor analysis highlights some new possible connections and relations among cytokines, which can show some further directions of investigation.

\section{Conclusions}

We conclude that our results indicate that complex and significant immunological mechanisms are associated with the pathogenesis of different forms of RD, such as RRD, PVR and PDR. Cytokines, chemokines and growth factors are upregulated in the vitreous of eyes with $R D$, and the upregulation is dependent on the form of RD. The detected proteins are present in different concentrations both in RRD and PVR. In the presence of PVR and PDR, the majority of cytokines are upregulated; thus, they may serve as biomarkers to estimate the progression or severity level of proliferation. Our study adds new biochemical information to previous studies in correlation with proliferative vitreoretinal alterations. More precise knowledge of the levels of vitreal cytokines may represent novel therapeutic targets in the management of these diseases. Future investigations should focus on identifying potential biomarkers to be able to intervene before irreversible proliferative alterations occur.

\section{Abbreviations}

CTACK: T-cell attracting chemokine; ELISA: Enzyme-linked immunosorbent assay; ERM: Epiretinal membrane; FGF: Fibroblast growth factor; G-

CSF: Granulocyte colony-stimulating factor; GM-CSF: Granulocytemacrophage colony-stimulating factor; GRO-alpha: Growth-related oncogene alpha; HGF: Hepatocyte growth factor; IFN: Interferon; IL: Interleukin; IL1ra: Interleukin-1 receptor antagonist; IL-2Ralpha: Interleukin-2 receptor alpha; IP-10: Interferon gamma-induced protein 10; LIF: Leukaemia inhibitory factor; MCP: Monocyte chemotactic protein; M-CSF: Macrophage colony-stimulating factor; MH: Macular hole; MIF: Macrophage migration inhibitory factor; MIG: Monokine induced by interferon gamma; MIP: Macrophage inflammatory protein; beta-NGF: Beta-nerve growth factor; PDGF-BB: Plateletderived growth factor; PDR: Proliferative diabetic retinopathy; PVR: Proliferative vitreoretinopathy; RANTES: Regulated upon activation, normal T cell expressed and secreted; RD: Retinal detachment; RRD: Rhegmatogenous retinal detachment; SCF: Stem cell factor; SDF1alpha: Stromal cell-derived factor 1alpha; SCGF-beta: Stem cell growth factor beta; TNF: Tumour necrosis factor; TRAIL: Tumour necrosis factorrelated apoptosis-inducing ligand; VEGF: Vascular endothelial growth factor 


\section{Acknowledgements}

Authors are grateful to Dariusz Pianka and Beáta Mátrai at Bio-Rad Laboratories and Barbara Pethes for their support in the analysis.

\section{Authors' contributions}

AB prepared the manuscript, analyzed and interpreted the patients' data. VSZ reviewed the paper, KK reviewed the paper and interpreted the patients' data. ZN was a contributor in writing the manuscript. MR, TM treated the patients, reviewed the manuscript. All authors read and approved the final manuscript.

\section{Authors' information}

Supported by the ÚNKP-19-3-I New National Excellence Program of the Ministry for Innovation and Technology."

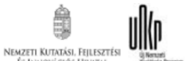

\section{Funding}

The authors declare that they have no funding.

\section{Availability of data and materials}

The datasets generated and/or analysed during the current study are not publicly available due further analysis of data is in progress, but are available from the corresponding author on reasonable request.

\section{Ethics approval and consent to participate}

The present study was approved by the Hungarian Medical Research Council Committee of Science and Research Ethics (Approval No. 15028-2/2017/EKU) and performed by following per under the tenets of the Declaration of Helsinki. All participants gave written informed consent to the study.

\section{Consent for publication}

Not applicable. No image or other personal or clinical details of participants are shown, which would compromise anonymity.

\section{Competing interests}

The authors declare that they have no competing interests.

\section{Author details}

'Department of Ophthalmology, Semmelweis University, Mária u 39, Budapest 1085, Hungary. ${ }^{2}$ Department of Ophthalmology, Uzsoki Hospital Budapest, Uzsoki u. 29-41, Budapest 1145, Hungary. ${ }^{3}$ Department of Ophthalmology, University of Eastern Finland and Kuopio University Hospital, Kuopio, Finland.

Received: 3 April 2020 Accepted: 25 November 2020

Published online: 28 December 2020

\section{References}

1. Garweg JG, Tappeiner C, Halberstadt M. Pathophysiology of proliferative vitreoretinopathy in retinal detachment. Surv Ophthalmol. 2013;58(4):321-9.

2. Wong TY, Cheung CM, Larsen M, Sharma S, Simo R. Diabetic retinopathy. Nat Rev Dis Primers. 2016:2:16012.

3. Khan MA, Brady CJ, Kaiser RS. Clinical management of proliferative vitreoretinopathy: an update. Retina (Philadelphia, Pa). 2015;35(2):165-75.

4. Iyer SSR, Regan KA, Burnham JM, Chen CJ. Surgical management of diabetic tractional retinal detachments. Surv Ophthalmol. 2019;64(6):780-809.

5. Pastor JC. Proliferative vitreoretinopathy: an overview. Surv Ophthalmol. 1998;43(1):3-18.

6. Kauffmann DJ, van Meurs JC, Mertens DA, Peperkamp E, Master C, Gerritsen ME. Cytokines in vitreous humor: interleukin- 6 is elevated in proliferative vitreoretinopathy. Invest Ophthalmol Vis Sci. 1994;35(3):900-6.

7. Elner SG, Elner VM, Jaffe GJ, Stuart A, Kunkel SL, Strieter RM. Cytokines in proliferative diabetic retinopathy and proliferative vitreoretinopathy. Cur Eye Res. 1995;14(11):1045-53.

8. Abu El-Asrar AM, Struyf S, Kangave D, Geboes K, Van Damme J. Chemokines in proliferative diabetic retinopathy and proliferative vitreoretinopathy. Eur Cytokine Netw. 2006;17(3):155-65.

9. Capeans C, De Rojas MV, Lojo S, Salorio MS. C-C chemokines in the vitreous of patients with proliferative vitreoretinopathy and proliferative diabetic retinopathy. Retina (Philadelphia, Pa). 1998;18(6):546-50.
10. Dai Y, Wu Z, Sheng H, Zhang Z, Yu M, Zhang Q. Identification of inflammatory mediators in patients with rhegmatogenous retinal detachment associated with choroidal detachment. Mol Vis. 2015;21:417-27.

11. Ricker $L$, Kessels AG, de Jager W, Hendrikse F, Kijlstra A, la Heij EC. Prediction of proliferative vitreoretinopathy after retinal detachment surgery: potential of biomarker profiling. Am J Ophthalmol. 2012;154(2):347-54.e2.

12. Takahashi S, Adachi K, Suzuki Y, Maeno A, Nakazawa M. Profiles of inflammatory cytokines in the vitreous fluid from patients with Rhegmatogenous retinal detachment and their correlations with clinical features. Biomed Res Int. 2016;2016:4256183

13. Kunikata H, Yasuda M, Aizawa N, Tanaka Y, Abe T, Nakazawa T. Intraocular concentrations of cytokines and chemokines in rhegmatogenous retina detachment and the effect of intravitreal triamcinolone acetonide. Am J Ophthalmol. 2013;155(6):1028 37.e1

14. Balogh A, Milibák T, Szabó V, Nagy ZZ, Resch MD. Position of macula lutea and presence of proliferative vitreoretinopathy affect vitreous cytokine expression in rhegmatogenous retinal detachment. PLoS One. 2020;15(6):e0234525.

15. Wladis EJ, Falk NS, Iglesias BV, Beer PM, Gosselin EJ. Analysis of the molecular biologic milieu of the vitreous in proliferative vitreoretinopathy. Retina (Philadelphia, Pa). 2013:33(4):807-11.

16. Roybal CN, Velez G, Toral MA, Tsang SH, Bassuk AG, Mahajan VB. Personalized proteomics in proliferative Vitreoretinopathy implicate hematopoietic cell recruitment and mTOR as a therapeutic target. Am J Ophthalmol. 2018:186:152-63.

17. Zhou P, Zhao MW, Li XX, Yu WZ, Bian ZM. siRNA targeting mammalian target of rapamycin (mTOR) attenuates experimental proliferative vitreoretinopathy. Curr Eye Res. 2007;32(11):973-84.

18. Pennock S, Kim D, Mukai S, Kuhnle M, Chun DW, Matsubara J, et al. Ranibizumab is a potential prophylaxis for proliferative vitreoretinopathy, a nonangiogenic blinding disease. Am J Pathol. 2013;182(5):1659-70.

19. Cui W, Zhang H, Liu ZL. Interleukin-6 receptor blockade suppresses subretinal fibrosis in a mouse model. Int J Ophthalmol. 2014;7(2):194-7.

20. Kawahara S, Hata Y, Kita T, Arita R, Miura M, Nakao S, et al. Potent inhibition of cicatricial contraction in proliferative vitreoretinal diseases by statins. Diabetes. 2008:57(10):2784-93.

21. Wang $Y$, Gao S, Zhu Y, Shen X. Elevated activating transcription factor 4 and glucose-regulated $78 \mathrm{Kda}$ protein levels correlate with inflammatory cytokines in the aqueous humor and vitreous of proliferative diabetic retinopathy. Curr Eye Res. 2017;42(8):1202-8.

22. Dai Y, Wu Z, Wang F, Zhang Z, Yu M. Identification of chemokines and growth factors in proliferative diabetic retinopathy vitreous. Biomed Res Int 2014:2014:486386.

23. Salam A, Mathew $R$, Sivaprasad S. Treatment of proliferative diabetic retinopathy with anti-VEGF agents. Acta Ophthalmol. 2011;89(5):405-11.

24. Song Z, Sun M, Zhou F, Huang F, Qu J, Chen D. Increased intravitreous interleukin-18 correlated to vascular endothelial growth factor in patients with active proliferative diabetic retinopathy. Graefes Arch Clin Exp Ophthalmol. 2014:252(8):1229-34.

25. Xu Y, Cheng Q, Yang B, Yu S, Xu F, Lu L, et al. Increased sCD200 levels in vitreous of patients with proliferative diabetic retinopathy and its correlation with VEGF and Proinflammatory cytokines. Invest Ophthalmol Vis Sci. 2015;56(11):6565-72.

26. Loukovaara S, Piippo N, Kinnunen K, Hytti M, Kaarniranta K, Kauppinen A. NLRP3 inflammasome activation is associated with proliferative diabetic retinopathy. Acta Ophthalmol. 2017;95(8):803-8.

27. Kaarniranta K, Salminen A. Age-related macular degeneration: activation of innate immunity system via pattern recognition receptors. J Mol Med (Berlin, Germany). 2009;87(2):117-23.

28. Pollreisz A, Sacu S, Eibenberger K, Funk M, Kivaranovic D, Zlabinger GJ, et al. Extent of detached retina and Lens status influence Intravitreal protein expression in Rhegmatogenous retinal detachment. Invest Ophthalmol Vis Sci. 2015:56(9):5493-502.

29. Rasier R, Gormus U, Artunay O, Yuzbasioglu E, Oncel M, Bahcecioglu H. Vitreous levels of VEGF, IL-8, and TNF-alpha in retinal detachment. Curr Eye Res. 2010;35(6):505-9.

30. Ricker L, Kijlstra A, Kessels AG, de Jager W, Liem AT, Hendrikse F, et al. Interleukin and growth factor levels in subretinal fluid in rhegmatogenous retinal detachment: a case-control study. PLoS One. 2011;6(4):e19141.

\section{Publisher's Note}

Springer Nature remains neutral with regard to jurisdictional claims in published maps and institutional affiliations. 